\title{
Two Electrons in Vertically Coupled One-Dimensional Rings
}

\author{
Jairo H. Marin ${ }^{1}$, Francisco García ${ }^{2}$, and Ilia D. Mikhailov ${ }^{2}$ \\ ${ }^{1}$ Escuela de Física, Universidad Nacional de Colombia, A. A. 3840 Medellín \\ ${ }^{2}$ Escuela de Física, Universidad Industrial de Santander, A. A. 678 Bucaramanga
}

\section{Received on 8 December, 2005}

\begin{abstract}
A problem of two electrons spatially separated in vertically coupled one-dimensional rings is solved exactly by using the numerical trigonometric sweep method. The change of the level-ordering and the crossover of the curves of the energy levels as a functions of the rings radii, the separation between rings and the magnetic field, applied along the axis, are found and discussed. As the distance between rings tends to zero our results are in an excellent agreement with those obtained previously for the single two-electron one-dimensional ring.
\end{abstract}

Keywords: Quantum rings; Two-electron energies; Trigonometric sweep method

\section{INTRODUCTION}

The rapid progress in crystal growth and fabrication methods have made possible to generate a new kind of nanometersize semiconductor heterostructures with controlled thickness and relatively sharp interfaces. In the last several years, there has been a considerable interest in quasi-zero dimensional self-assembled quantum dots (QDs), formed through the Stranki-Krastanow growth mode by deposition a material on the substrate with different lattice parameter [1]. This interest is related to potential technological application of QDs in optoelectronic devices [1-2], as well as to their character of novel quantum systems in which new physics and analogies with atomic clusters, atoms, molecules, may be found. The QDs shapes are not well known yet, nevertheless, structural studies suggest that commonly the quantum dots look like lenses, pyramids or disks [3]. Recently, Lorke and co-workers have reported the fabrication of InGaAs QDs with a ring-like geometry [4]. This finding has stimulated some theoretical investigations about one- and two-particle spectra in quantum rings (QRs) which have been done in order to establish the peculiarities related to this morphology [5]. The realistic geometry of two-electron InGaAs QR is a structure with larger outer radius $(\sim 400-500 \mathrm{~nm})$, narrower width $(\sim 20-40 \mathrm{~nm})$ and very small height $(\sim 2-4 \mathrm{~nm})$. Due to strong confinement in vertical direction a two-dimensional model is commonly considered in order to analyze the QRs electronic properties. Nevertheless, one-dimensional model may also be consider as appropriated in framework of the adiabatic approximation [5] which allows one to decouple the rapid radial motion from the slow angular motion.

Exact energy levels of two-electron systems in 1D QRs have been obtained and the size effect on the interaction energies in the limit of a narrow-width nanoring has been studied in reference [6]. A few two-particle exactly solvable problems are known and therefore the exact solution found out in reference [6] is interesting not only from a physical point of view but also from a mathematical point of view. In this work we propose an other exactly solvable model in which two vertically coupled concentric 1D rings contain each one only one electron. The fabrication of similar stacked arrays of InAs self-assembled QDs with vertical alignment have been reported recently [7]. These systems are technologically im- portant, because they allow us to improve the performance of the optical devices taking advantage provided by the coupling effect between QRs. In this context, we consider relevant to use the exactly solvable model to explore the new physics bound up with the coupled QRs in order to understand effects related to coupling between rings on the two-electron energy spectrum.

\section{THEORY}

We consider a model of two coaxial, identical and parallel one-dimensional rings, every each with only one electron. Besides, we suppose that the external homogeneous magnetic field $\mathbf{B}=B \hat{\mathbf{z}}$ is applied along the rings axis. Being $R$ the rings radius and $d$ the spatial separation between them along the Zdirection, we introduce dimensionless parameter $\beta=d / R$. In order to compare our results with those from Ref. 6 in the limit case $\beta \rightarrow 0$, as rings merge and form only one ring, we use both the same notations and the units that have been used in this reference. The units used below for the length, the energy and the magnetic field strength are the effective Bohr radius, $a_{0} *=\hbar^{2} \varepsilon / m * e^{2}$, the effective Rydberg $R y *=m * e^{4} / 2 \hbar^{2} \varepsilon^{2}$ and the first Landau level energy $\gamma=e B \hbar / 2 m^{*} c R y^{*}$, respectively.

The normalized dimensionless two-electron Hamiltonian $\left(\tilde{H}=H R^{2}\right)$ :

$$
\begin{array}{r}
\tilde{H}=\sum_{i=1}^{2}\left[-\frac{\partial^{2}}{\partial \varphi_{i}^{2}}-i \gamma R^{2} \frac{\partial}{\partial \varphi_{i}}\right]+\frac{\gamma^{2} R^{4}}{2}+U\left(\varphi_{1}-\varphi_{2}\right) \\
U\left(\varphi_{1}-\varphi_{2}\right)=2 R / \sqrt{4 \operatorname{sen}^{2}\left(\frac{\varphi_{1}-\varphi_{2}}{2}\right)+\beta^{2}}
\end{array}
$$

Eigenvalues of the Hamiltonian (1) corresponding to the normalized two-electron energies, $\tilde{E}$ (energies $E$ multiplied by the squared radius of ring, $\tilde{E}=E R^{2}$ ) can be found exactly by using center-of-mass, $\theta=\left(\varphi_{1}+\varphi_{2}\right) / 2$ and relative, $\varphi=\varphi_{1}-\varphi_{2}$ coordinates. In these coordinates, the Hamiltonian (1) can be separated into center-of-mass, $\tilde{H}_{c}$ and relative, $\tilde{H}_{r}$ terms: 


$$
\begin{aligned}
& \tilde{H}=\tilde{H}_{c}+\tilde{H}_{r}+\gamma^{2} R^{4} / 2 ; \quad \tilde{H}_{c}=-\frac{1}{2} \frac{d^{2}}{d \theta^{2}}-i \gamma R^{2} \frac{d}{d \theta} \\
& \tilde{H}_{r}=-2 \frac{d^{2}}{d \varphi^{2}}+U(\varphi)
\end{aligned}
$$

This separability allows us to write two-particle normalized energy in the form $\tilde{E}=\tilde{E}_{c}+\tilde{E}_{r}+\gamma^{2} R^{4} / 2$, where $\tilde{E}_{c}$ and $\tilde{E}_{r}$ are eigenvalues of the operators $\tilde{H}_{c}$ and $\tilde{H}_{r}$, respectively. The eigenvalues of the operator $\tilde{H}_{c}$ are given by: $\tilde{E}_{c}(M)=$ $0.5 M^{2}+\gamma M R^{2}$ with center-of-mass angular momentum $M=$ $0, \pm 1, \pm 2, \ldots$, whereas the eigenvalues of the operator $\tilde{H}_{r}$ should be found by solving the one-dimensional wave equation:

$$
-2 \frac{d^{2} f_{m s}(\varphi)}{d \varphi^{2}}+U(\varphi) f_{m s}(\varphi)=\tilde{E}_{r}(m, s) f_{m s}(\varphi)
$$

in the region of $[-2 \pi, 2 \pi]$ with periodic boundary conditions $f_{m s}(0)=(-1)^{m} f_{m s}(2 \pi)$. The quantum number $m=$ $0, \pm 1, \pm 2, \ldots$ defines the two-electron relative angular momentum and $s= \pm$ the eigenfunction parity. The even solutions $(s=+)$ correspond to singlet states and the odd solutions $(s=-)$ correspond to triplet states. The electronic spectrum of the two-electron system will then be given by energies $\tilde{E}(M, m, s)$ labelled by three quantum numbers $M, m, s$. In our numerical work we use the trigonometric sweep method [8] in order to solve the eigenvalue problem (4) with the periodic boundary condition asserted above.

\section{RESULTS}

We have performed numerical calculations of two-electron energies $\tilde{E}(M, m, \pm)$ as a function of the rings radius $R$ for different values of $\beta$. The results are presented in Fig.1 and Table I. Here and below we use the short notation about quantum number and spin, i.e. $a, b, c$, etc. in the order of increasing energy value under very strong confinement condition $(R \rightarrow 0)$ as the rings merge $(\beta \rightarrow 0)$.

Complete correspondence between short notation and set of the quantum numbers $(M, m, s)$ is given in Table I. In the same place we compare our results for very small ratio $\beta=0.001$ with those obtained previously for two electrons in a single ring in Ref. 6. One can observe an excellent concordance between results obtained for two different rings radii.

One can observe in Fig.1 a remarkable evolution of the energies dependencies on the ring radius with increasing of the separation between rings, as the ratio $\beta$ varies from small value 0,001 (upper curves) to large one 10 (lower curves). To understand this evolution one should compare the contributions which give the kinetic and Coulomb energies in the Hamiltonian (1). There is a strong competition between these two terms. As the ring radius is very small or the distance between rings is very large the contribution of the Coulomb energy becomes depreciable and the exact eigenvalues of the Hamiltonian (1) correspond to normalized energies of two uncoupled rigid rotors given by the formula:
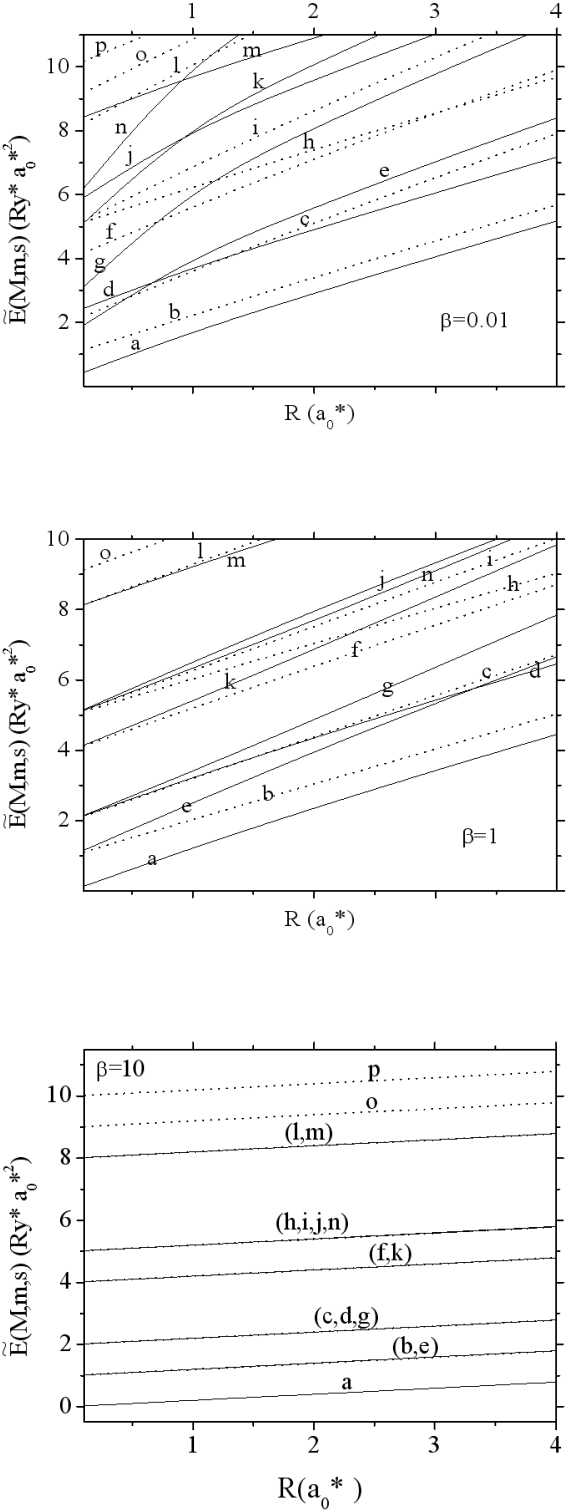

FIG. 1: Normalized total energies $\tilde{E}(M, m, s)$ of two electrons versus radius $R$ in vertically coupled QRs with different ratios of the distance between rings-to-radius, $\beta$ 0.001, 1 and 10. Solid lines are singlet states and dotted lines are triplet states.

$$
\tilde{E}(M, m, s)=0.5\left(M^{2}+m^{2}\right)
$$

It is seen from Fig. 1 that the energies fulfilled this relation for $R \rightarrow 0$ and for all values of $\beta$ both for singlet and triplet states. As the rings radii increases the contribution of the Coulomb energy grows and one can observe intersections of higher levels with same or different spin induced by the electron-electron interaction. The number of the intersections substantially decreases with increasing the ratio $\beta$ and when the value of $\beta$ is very large the curves of the energies depen- 
TABLE I: Exact energy levels $\tilde{E}(M, m, s)$ of two electrons in vertically coupled rings with $\beta=0.001$ (our results) and in single ring (from Ref. 6).

\begin{tabular}{|l|l|l|l|l|l|}
\hline \multicolumn{2}{|l|}{ Energy notation } & \multicolumn{2}{l|}{$R=1 a_{0} *$} & \multicolumn{2}{l|}{$R=4 a_{0} *$} \\
\hline $\begin{array}{l}\text { Short no- } \\
\text { tation }\end{array}$ & $\begin{array}{l}\text { Full nota- } \\
\text { tion }\end{array}$ & $\begin{array}{l}\text { Our } \\
\text { results }\end{array}$ & Ref. 6 & $\begin{array}{l}\text { Our } \\
\text { results }\end{array}$ & Ref.6 \\
\hline $\mathrm{a}$ & $\tilde{E}(0,0,0)$ & 1.72 & 1.73 & 5.18 & 5.18 \\
\hline $\mathrm{b}$ & $\tilde{E}(1,1,1)$ & 2.23 & 2.23 & 5.68 & 5.68 \\
\hline $\mathrm{c}$ & $\tilde{E}(0,2,1)$ & 3.61 & 3.62 & 7.92 & 7.92 \\
\hline $\mathrm{d}$ & $\tilde{E}(2,0,0)$ & 3.72 & 3.73 & 7.18 & 7.18 \\
\hline $\mathrm{e}$ & $\tilde{E}(1,1,0)$ & 4.06 & 4.12 & 8.41 & 8.42 \\
\hline $\mathrm{f}$ & $\tilde{E}(2,2,1)$ & 5.62 & 5.62 & 9.92 & 9.92 \\
\hline $\mathrm{g}$ & $\tilde{E}(0,2,0)$ & 6.22 & 6.23 & 11.40 & 11.40 \\
\hline $\mathrm{h}$ & $\tilde{E}(3,1,1)$ & 6.33 & 6.38 & 9.68 & 9.68 \\
\hline $\mathrm{i}$ & $\tilde{E}(1,3,1)$ & 6.88 & 6.88 & 11.90 & 11.90 \\
\hline $\mathrm{j}$ & $\tilde{E}(3,1,0)$ & 8.06 & 8.11 & 12.41 & 12.41 \\
\hline $\mathrm{k}$ & $\tilde{E}(2,2,0)$ & 8.22 & 8.38 & 13.40 & 13.40 \\
\hline 1 & $\tilde{E}(0,4,1)$ & 10.08 & 10.08 & 15.71 & 15.71 \\
\hline $\mathrm{m}$ & $\tilde{E}(4,0,0)$ & 9.72 & 9.73 & 13.18 & 13.18 \\
\hline $\mathrm{n}$ & $\tilde{E}(1,3,0)$ & 10.24 & 10.57 & 16.19 & 16.21 \\
\hline $\mathrm{o}$ & $\tilde{E}(3,3,1)$ & 10.88 & 10.88 & 15.90 & 15.90 \\
\hline $\mathrm{p}$ & $\tilde{E}(4,2,1)$ & 11.62 & 11.62 & 15.92 & 15.92 \\
\hline
\end{tabular}

dencies on the ring radius become almost horizontal and the levels turn into degenerated with respect to spin orientations.

Similar dependencies of the two electron energies on the distance between vertically coupled one-dimensional rings are presented in Fig. 2 for ring radii $1 a_{0} *$ and $5 a_{0} *$. One can observe that the spectrum of the system substantially transforms to one, typical for a pair of uncoupled rigid rotors described by the relation (5). Transformation of the energy spectrum from one common for a system with a strong Coulomb interaction (as the distance between rings is small) to another usual for a system of the independent rigid rotors (as the distance between rings is large) provides the intersections between different curves observed in Fig. 2 .

The dependence of some normalize energies $\tilde{E}( \pm M, m, s)$ with $|M| \leq 2$ on the magnetic field strength for the ring radii $R=1 a_{0} *$, and separation between rings, $d=0.1 a_{0} *$, is shown in Fig. 3. One can see that the presence of the magnetic field provides the splitting of the energy levels whose angular momentum $M$ has different signs. Larger magnetic field strength more significant is the splitting induced by the paramagnetic term in the Hamiltonian (3).

The behaviour of these curves is controlled by the strong competition between the paramagnetic and diamagnetic terms in the Hamiltonian (3). The contribution of the paramagnetic term depends on the magnetic field strength is linear whereas this dependence of the diamagnetic term is quadratic. Therefore, as the magnetic field strength is small the contribution of the paramagnetic term is predominant and for large magnetic field the diamagnetic term becomes more significant. For this reason, at the beginning of the curves they look like quasilinear with a positive slope $(M \geq 0)$ or negative slope $(M<0)$
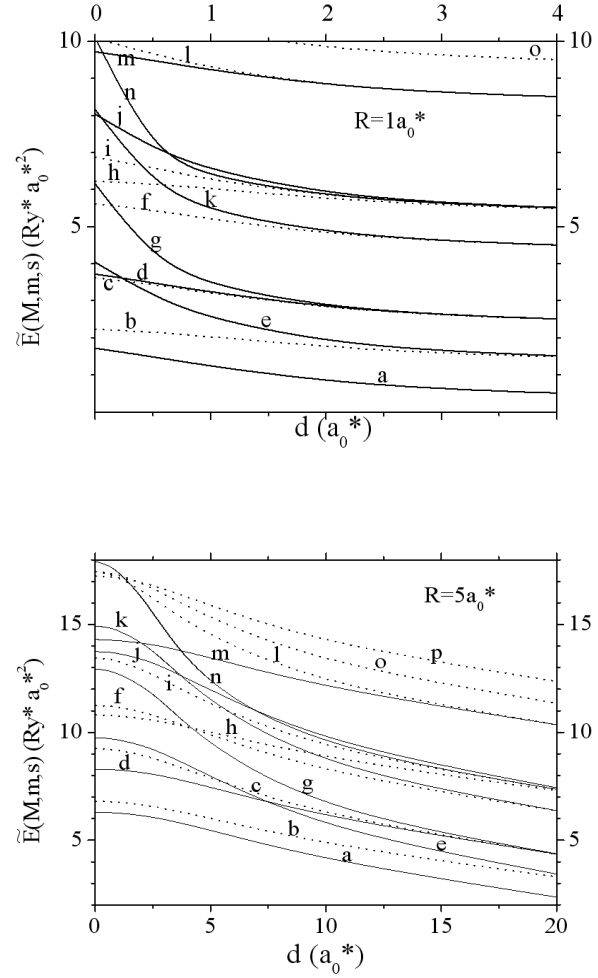

FIG. 2: Normalized total energies $\tilde{E}(M, m, s)$ of two electrons versus distance $d$ between vertically coupled rings with two different radii, $R=1 a_{0} *$ (upper curves) and $R=5 a_{0} *$ (lower curves).

adopting parabolic-like shape for large magnetic fields. Additionally, we can note that the lowest level energy dependence on the magnetic field strength display an oscillatory behaviour with period $\gamma=1.0$. For magnetic field strength less than 0.5 the ground state energy corresponds to the $\tilde{E}(0,0,0)$ singlet level. As the magnetic field strength becomes greater than 0.5 the ground state is transformed in $\tilde{E}(-1,1,1)$ triplet level. Within the interval of the magnetic field $(1<\gamma<1.5)$ this level is remained as the ground state. Further, as the magnetic field exceeds the value 1.5 the ground state is transformed in $\tilde{E}(-2,0,0)$ singlet level, etc. This oscillatory behaviour is remained for different values of the rings radius and the separation between them. It is evident that the quantum number $M$ of the ground state level raises with increasing of the magnetic field strength and the positions of minima in the curves of the magnetic oscillation corresponds to $\gamma=M / R^{2}$ and the oscillation period is equal to $1 / R^{2}$. Magnetic oscillations not suffer any changes as the ring radii are modified. It is due to the fact that the Coulomb interaction between two electrons in $1 \mathrm{D}$ rings is responsible of the quantum size effects.

In conclusion, we consider a new exactly solvable twoelectron model, which describes the energy spectrum of two vertically coupled one-dimensional rings. The energy levels dependencies on the rings radii, the separation between rings and the strength of the magnetic field applied parallel to the 


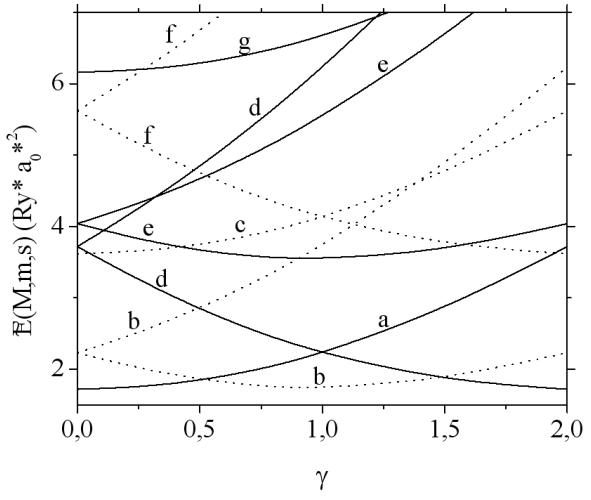

FIG. 3: Normalized energies $\tilde{E}( \pm M, m, s)$ versus magnetic field strength $\gamma$ for two electrons in vertically coupled rings of the radii $R=1 a_{0} *$ and separation between them $d=0.1 a_{0} *$. axis are analyzed. It is shown that in the limit case, when the separation between rings tends to zero, our results are in an excellent concordance with those obtained previously for one-dimensional ring by using a different method.

\section{Acknowledgements}

This work was financed by the Industrial University of Santander (UIS) through the Dirección General de Investigaciones (DIF Ciencias, Cod. Cod. 5124) and the Excellence Center of Novel Materials ECNM, under Contract No. 043-2005 and the Cod. No. 1102-05-16923 subscribed with COLCIENCIAS. J. H. Marín wishes to thank the Universidad Nacional-Sede Medellín for the permission to study at the UIS.
[1] L. Jacak, P. Hawrylak, and A. Wójs, Quantum Dots (Springer, Berlin, 1997).

[2] G. Timp, Nanotechnology (Springer-Verlag, New York, 1999).

[3] S. Fafard, Appl. Phys. Lett. 76, 2707 (2000).

[4] A. Lorke, R. J. Luyken, A. O. Govorov, J. P. Kotthaus, J. M. Garcia, and P. M. Petroff, Phys. Rev. Lett. 84, 2223 (2000).

[5] H. Hu, J. L. Zhu, and J. J. Xiong, Phys. Rev. B 62, 16777 (2000); L. Wendler, V. M. Fomin, A. V. Chaplik, and A. O. Govorov,
Phys. Rev. B 54, 4794 (1996).

[6] J.-L. Zhu, Z. Dai, and X. Hu, Phys. Rev. B 68, 045324 (2003).

[7] J. J Palacios and P. Hawrylak, Phys. Rev. B 51, 1769 (1995); G. S. Solomon, J. A. Trezza, A. F. Marshall, and J. S. Harris, Jr., Phys. Rev. Lett. 76, 952 (1996).

[8] F. J. Betancur, I. D. Mikhailov, and L. E. Oliveira, J. Appl. Phys. D 31, 3391 (1998). 